Www.jmscr.igmpublication.org

Impact Factor 5.244

Index Copernicus Value: 83.27

ISSN (e)-2347-176x ISSN (p) 2455-0450

crossref DOI: _https://dx.doi.org/10.18535/jmscr/v4i11.68

\title{
The Functional Outcome of Management of Acute Acromioclavicular Joint Disruption Using Tension Band Wiring
}

\author{
Authors
}

\author{
Dr P.S.T.Jambukeswaran ${ }^{1}$, Prof Dr A.Senthilnathan ${ }^{2}$, Dr R.Prabhakar ${ }^{3}$ \\ ${ }^{* 1}$ Post Graduate - Department of Orthopaedics, RMMCH, Annamalai University \\ ${ }^{2}$ Professor - Department of Orthopaedics, RMMCH, Annamalai University \\ ${ }^{3}$ Registrar - Department of Orthopaedics, RMMCH, Annamalai University
}

\section{ABSTRACT}

Injuries to the acromioclavicular (AC) joint represent a spectrum of soft tissue disruptions that can result in mild, transient pain to significant displacement, chronic pain \& changes in shoulder biomechanics resulting in longterm disability. The aim of our study was mainly to analyze the functional outcome of complete acromioclavicular injuries treated with $K$ wire and Tension Band Wiring. 20 cases of acromioclavicular disruption (type III - V) were treated with open reduction and tension band wiring in Division of Orthopaedics, Rajah Muthiah Medical College, Annamalai University. Closed acute complete acromioclavicular joint disruptions (Rockwood and Young type III-VI) between the age group of 18-60 years were included in the study. Chronic disruption \& compound injuries were excluded from the study. After obtaining informed consent and anaesthetic fitness, open reduction \& tension band wiring was done. Post operative X-rays assessed. All patients underwent a standardized post operative protocol with imobilisation in strapping for 3 weeks. After which shoulder mobilization started. Functional outcome assessed at regular interval using DASH \& CONSTANT scores.

RESULTS: At the last follow-up, 18 patients had an excellent outcome as assessed by Constant score, DASH scores. One patients had good outcome. One patient had fair outcome. CONCLUSION: Tension band wiring in acute complete Acromioclavicular injuries has resulted in excellent functional outcome. Though there is risk of implant breakage and need for implant removal at later stage, this technique has the advantage of being cost effective, short surgical time and early rehabilitation.

KEY WORDS: DASH-The Disabilities of the Arm Shoulder and Hand Score.

\section{INTRODUCTION}

Acromioclavicular (AC) joint injuries account for approximately $9 \%$ of shoulder girdle. Injuries to the acromioclavicular (AC) joint represent a spectrum of soft tissue disruptions that can result in mild, transient pain to significant displacement, chronic pain \& changes in shoulder biomechanics results in longterm disability. In AC injures males are affected most commonly with a male-tofemale ratio of approximately 5:1 \& age group affected $<30$ years and are commonly occurs in athletes and contact sport persons in which the mechanism of injury is direct blow to the lateral aspect of shoulder .

Management of Acromioclavicular joint injury have a debate from the time of Hippocrates and Galen, regarding when operative management is necessary and which procedure produces the best functional outcome with least morbidity. It is accepted that conservative management is 
indicated for type I and II(Incomplete dislocation) AC injuries (Rockwood classification)2, for type IV,V,VI injuries (more severe, high velocity injury) most surgeons agree that the displacement of AC joint would be too great to accept and operative modality is indicated but there is still controversy exists for the management of Type III injuries (complete dislocation).

The aim of this study is to analyze the functional outcome of reconstructions done for complete acromioclavicular joint disruptions using $\mathrm{k}$ wires $\&$ tension band wiring $\&$ assess the reduction and ac joint stability clinically and radiologically.

\section{MATERIALS AND METHODS}

This is a prospective study conducted in Rajah Muthiah Medical College Hospital, Annamalai University, during the period between July 2015 to September 2016 of 20 cases of complete Acromioclavicular Joint injuries (Rockwood type III-V) treated by K Wire \& Tension band Wiring. Patients with closed, acute, complete acromioclavicular joint disruptions (Rockwood and Young type III-VI) in the age group of 18-60years were included in the study Chronic injuries, elderly patients and open injuries were excluded from this study.

\section{CLINICAL EXAMINATION}

All patients received in the emergency ward were evaluated for any associated major injuries like chest injury, brachial plexus injury. On clinical evaluation patients presented with local tenderness, prominent lateral end of clavicle, local bruising, and ballotability.

\section{RADIOGRAPHIC EVALUATION}

Radiographic evaluation was done by taking 3 views: 1. Antero-posterior view (10 degree cephalad view - Zanca view) for both AC joint sitting or standing. Complete dislocation was diagnosed if lateral end of clavicle was elevated by atleast $75 \%$ of width of articular surface of acromial process. 2. Axillary lateral view to visualize anterior or posterior displacement of clavicle. 3.Stress view with $5 \mathrm{~kg}$ weight in both hands when patient is standing upright and pulling back his/her shoulders.

After stabilizing the general condition, and obtaining anaesthetic fitness, patient was taken up for surgery after informed consent. All cases were done under GA/RA.

\section{SURGICAL TECHNIQUE}

The angle of acromium is palpated and an incision 2 inch above is made horizontally extending to the anterior edge of the distal clavicle. Flaps are raised. Articular disc of AC JOINT was debrided to allow for good reduction. Manual reduction of clavicle is done and the reduction is held while from the top of the clavicle about $3 \mathrm{~cm}$ medially to the $\mathrm{AC}$ joint and midway between the anterior border and posterior border of the clavicle. AC dislocation reduced and held in position with two smooth $1.5 \mathrm{~mm} \mathrm{~K}$-wire (Intra-articular from lateral aspect of acromium to clavicle) under image intensifier control, followed by tension band wiring with no.18/20 stainless steel wire (316L).wound was closed. The arm was immobilized with jones strapping and arm sling.

\section{SURGICAL TECHNIQUE}
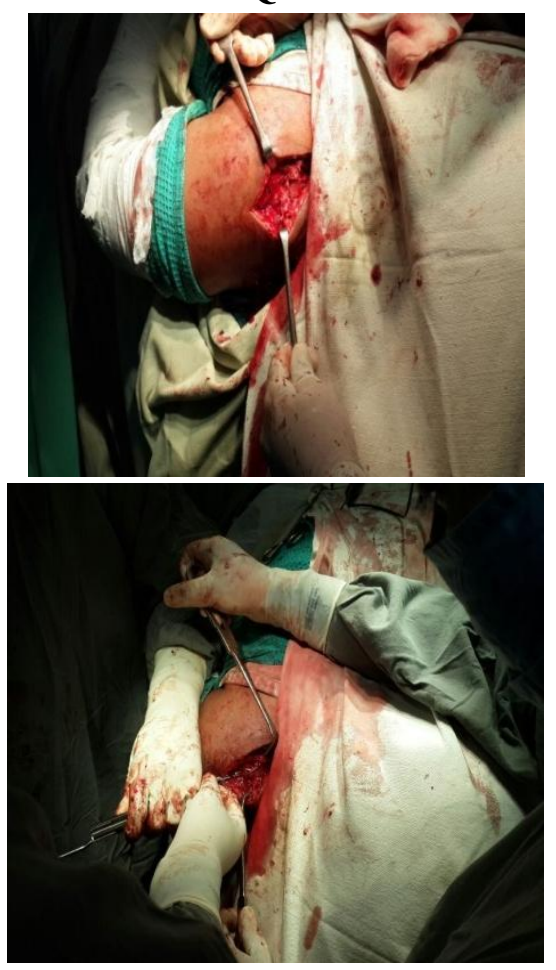


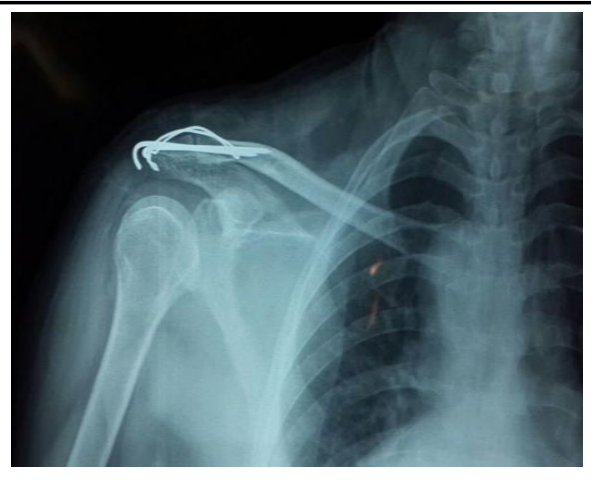

CASE ILLUSTRATION
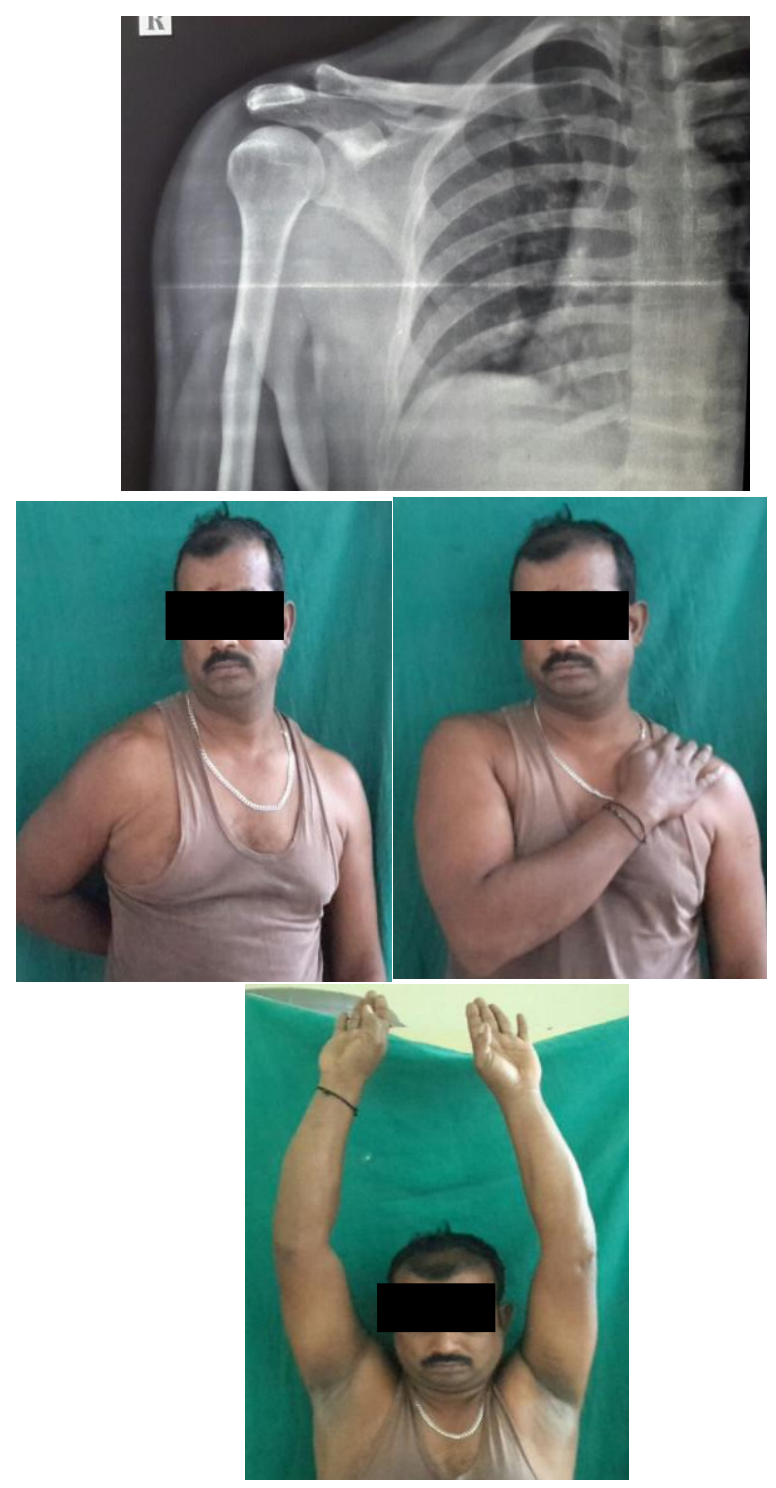

\section{POSTOPERATIVE PROTOCOL}

The surgical procedure described above took about 40 minutes for completion. Pendulum exercises were started on the 2 nd post operative date and passive mobilization started as patient tolerated. Within 3 weeks active exercises were started and full range of movement was started after 3 weeks.

We have used the DASH questionnaire and Constant score as they reflect the subjective and objective perspective of the shoulder function. The range of movement as required in the Constant score was measured with a goniometer.

\section{RESULTS}

We have done 20 cases of complete AC joint injuries during the period of July 2015 September 2016. We had 9 cases of Rockwood type 5, 6 cases of type 4, 5 cases of type 3 . We had 18 male cases, 2 female cases.

All 20 cases were closed injuries. Most common mode of injury is RTA. We had 6 right sided cases and 14 left sided cases. Most common associated injury is chest injury. The youngest patient in our study is 21 years and oldest is 45 years.

All cases were evaluated with $\mathrm{x}$-ray shoulder zanca view and $\mathrm{x}$-ray both shoulder AP stress view, Routine blood investigation and ECG was done.

Preoperative antibiotics were given. All injuries opened with horizontal lateral incision followed by reduction of $\mathrm{AC}$ joint and fixation with $\mathrm{K}$ wire \& TBW.

Average operating time was 40 minutes. Final fixatioin was checked under c-arm. Duration of postoperative stay is 8-10 days and all cases are started with pendulum exercises on the second postoperative day. Postop x-ray were taken. Postop outcome of reconstruction is measured by DASH Score, Constant score. Active full shoulder movements, active range of movements were started on 4 weeks.

Post operative complications were stitch granuloma - 1 case, Shoulder stiffness-1 case, superficial infection-1 case.

At the last follow-up, 18 patients had an excellent outcome as assessed by Constant score, DASH scores. One patients had good outcome. One patient had fair outcome. The mean scores at the last follow-up were: 
Constant score was 96 (range 80 -100), DASH score was 5.3 (range 1-11)

No vascular or neurological complications were noted. None of them had any functional deficits. Constant score is obtained from subjective and objective scoring including pain, activities of daily living, range of movement and muscle power. Excellent score is 100 and zero indicating poor score.
DASH questionnaire has 30 questions to be answered by the patient relating to activities of daily living, pain and confidence. Poorest outcome is 100 while the best outcome is a score of zero.

In our study outcome of reconstruction is studied extensively from operation table to full functional outcome till 6 months of followup.

\section{FUNCTIONAL OUTCOME}
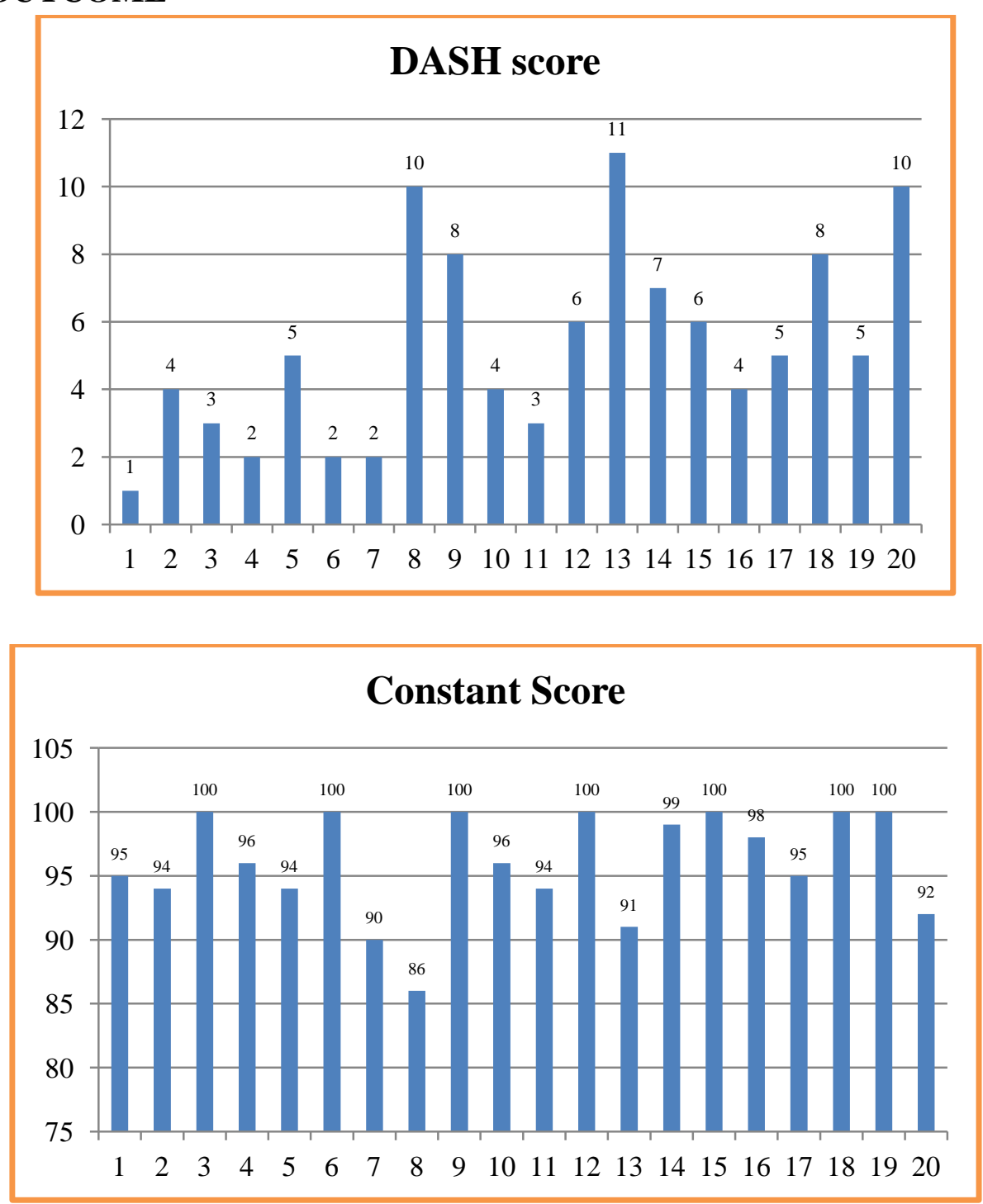

\section{DISCUSSION}

Acromioclavicular (AC) joint injuries account for approximately $9 \%$ of shoulder girdle injuries. Injuries to the acromioclavicular (AC) joint represent a spectrum of soft tissue disruptions that can result in mild, transient pain to significant displacement, chronic pain \& changes in shoulder biomechanics results in longterm disability. In AC injures males are affected most commonly with a male-to-female ratio of approximately 5:1 \& age group affected $<30$ years and are commonly occurs in athletes and contact sport persons in which the mechanism of injury is direct blow to the lateral aspect of shoulder. Management of Acromioclavicular joint injury have a debate from 
the time of Hippocrates and Galen, regarding when operative management is necessary and which procedure produces the best functional outcome with least morbidity.

There is still considerable controversy as to best method of managing AC dislocation. Conclusion drawn lot of variations as regard the study group, patient activity requirement, surgical expertise, type of fixation / repair / reconstruction, environmental factors etc. Conservative management of strapping / bracing/ splinting gives a 17 to $28 \%$ incidence of painful sequel due to meniscal damage, interposition of fibrous tissue or persistent instability. Disadvantages of conservative methods includes skin pressure sores and ulceration, necessity of wearing sling/brace for 8 weeks, poor patient compliance, interference with activities of daily living, no guarantee of freedom from pain, loss of shoulder and elbow motions, soft tissue calcifications, late $\mathrm{AC}$ joint arthritis, recurrence of deformity, late muscle atrophy, weakness and fatigue. If reconstructive procedure is required, then it's more difficult to perform later.

Surgical methods allow for inspection of AC joint, reduction of joint under vision, removal of damaged meniscus or loose fragments, repair of tear in capsule/ delto-trapezial Fascia / muscles. Surgical methods also permit an anatomical reduction and secure fixation that usually allows the resumption of shoulder motion earlier than is possible with closed techniques. Most other surgical methods also leave 9 to $28 \%$ patients with residual pain, sometimes due to complications of osteosynthesis. We consider that early AC degeneration is not caused by articular perforation with wires; it is also seen after conservative treatment and coracoclavicular fixation

In a study "Management of Acute Type III AC Dislocations- A study of 21 patients; managed by single technique- K-wire and tension band wiring along with repair of coraco-clavicular ligament" journal of trauma \& orthopaedics $2014 ; 9(4) ; 13$ 17 concluded that "Fixation with $\mathrm{K}$-wires and
Tension band wiring is a simple, easy, less time consuming surgical technique allowing faster rehabilitation in young and adult active patients to achieve a stable, pain free shoulder with no serious intra-operative or post-operative complications."

In an another study "Surgical treatment of acute complete acromioclavicular dislocation: comparison of tension band wiring with ligament transfer" among Twenty-nine patients with tension band wiring (group 1) and 27 patients with CA ligament reconstruction (group 2) were followed-up for 12-47 (mean, 23.6) months concluded that acute complete AC dislocation, treated with tension band wiring for the $\mathrm{AC}$ joint or CA ligament reconstruction achieved similar satisfactory rates. However, patients who received the former had relatively shorter operating times.

In another study "Comparison between Two Surgical Techniques Acromioclavicular Tension Band Wiring and Coracoclavicular Screw in Acromioclavicular Dislocations". Concluded that Bosworth screw and Tension band wiring are both useful procedures in patients with ACJ dislocation, but each should be used in the selected patients with special indications. Both methods had good results during follow-up period. There was not statistically meaningful difference in the articular stability, range of motion, OSS score and VAST SCORE between the two groups $(\mathrm{P}>0.05)$.

Most of the operative procedures for $\mathrm{AC}$ dislocation that have been reported had high incidence of such complications as breakage or migration of metallic device, failure of fixation or erosion of bone and subsequent loss of reduction. These difficulties may result in re-dislocation, infection and prolonged rehabilitation. Tension band wiring along with two smooth $1.5 \mathrm{~mm} \mathrm{~K}$ wires prevents the migration of $\mathrm{K}$-wires and gives enough stability to allow early mobilization. Smaller size or threaded K-wires were avoided as they have lesser strength so chances of breakage of wires at the joint are high. If larger dimension wires (more than $2 \mathrm{~mm}$ ) are used, they lead to 
osteolysis of lateral end of clavicle in certain cases. So we preferred K-wire of size $1.5 . \mathrm{mm}$ in all our 20 Patients. Most patients have no difficulty with activities of daily living but manual labourers (on some overhead activities) and athletes occasionally report pain with throwing and contact sports.

Our surgical technique gives comparable results with minimal complications and that there is no statistically significant difference in our outcomes as compared to other studies of AC fixation. The limitations of our study are that we have not evaluated the results in teenagers (immature skeleton) or geriatric (osteoporosis, less activity demand) age group patients or chronic dislocations or after failure of conservative treatment.

\section{CONCLUSION}

Tension band wiring in acute complete Acromioclavicular injuries has resulted in excellent functional outcome. Though there is risk of implant migration and need for implant removal at later stage, this technique has the advantage of being cost effective, short surgical time and early rehabilitation.

\section{REFERENCES}

1. Bucholz, Robert W.; Heckman, James D.; Court-Brown, Charles M. Rockwood \& Green's Fractures in Adults, 6th Edition. Volume2, Chapter 34 - Subluxations and Dislocations About the Glenohumeral Joint, Pages-1286 to 1299. Lippincott Williams \& Wilkins; 2006.

2. CemZeki ESENYEL, Kahraman ÖZTÜRK, Murat BÜLBÜL, Semih AYANOĞLU, Hasan Hüseyin CEYLAN. Coracoclavicular ligament repair and screw fixation in acromioclavicular dislocations. Acta Orthop Traumatol Turc 2010; 44(3): 194-198.

3. Cote MP, Wojcik KE, Gomlinski G, Mazzocca AD. Rebabilitation of acromioclavicular joint separations: operative and nonoperative considerations. Clin Sports Med. 2010 Apr; 29(2): 213-28, vii.

4. Esenyel $\mathrm{CZ}$, Oztürk $\mathrm{K}$, Bülbül $\mathrm{M}$, Ayanoğlu S, Ceylan HH. Coracoclavicular ligament repair and screw fixation in acromioclavicular dislocations. Acta Orthop Traumatol Turc 2010; 44:194-198.

5. Imatani RJ, Hanlon JJ, Cady GW. Acute, Complete acromioclavicular separation. J Bone Joint Surg [Am] 1975; 57:328-32.

6. Lemos MJ, Tolo ET. Complications of treatment of the acromioclavicular and sternoclavicular joint injuries, including instability. Clin sports Med. 2003 Apr; 22(2):371-85.

7. Milewski MD, Tompkins M, Giugale JM, et al. Complications related to anatomic reconstruction of the Coracoclavicular ligaments. Am J Sports Med 2012; 40:1628-1634.

8. Simovitcb R, Sanders B, Ozbaydar M, et al. Acromioclavicular joint injuries: diagnosis and management. J Am AcadOrtbop Surg.2009 Apr; 17(4):207-19.

9. Soni RK. Conservatively treated acromioclavicular joint dislocation: a 45years follow-up. Injury 2004; 35: 548-50.

10. Suezie Kim, M.D., L.T., M.C., U.S.N., Alan Blank, M.D., and Eric Strauss, M.D. Management of Type 3 Acromioclavicular Joint Dislocations-Current Controversies. Bulletin of the Hospital for Joint Diseases 2014;72 (1) :53-60

11. Wei-Ching Lin, CHI-Chaun Wu, Chun-Yi $\mathrm{Su}$, Kuo-Feng Fan, I-Chaun Tseng, Yi-Lee Chiu. Surgical Treatment of Acute Complete Acromioclavicular Dislocation: Comparison of Coracoclavicular Screw Fixation Supplemented with Tension Band Wiring or Ligament Transfer. Clin Sports Med. 2008 Aug; 29(2): 213-28. 